\title{
Selection of sensory information in control of pursuit eye movements'
}

ADAM ATKIN

DEPT. OF NEUROLOGY, THE MOUNT SINAI HOSPITAL, NEW YORK CITY

Ss performed fixation shifts between moving and stationary targets that were presented against a visually complex background. Post-saccade pursuit latencies were far shorter than the response lags previously reported for the pursuit control system. This is interpreted as evidence for control of initial post-saccade pursuit velocity by pre-saccade image velocity information selected from a limited region of peripheral retina.

In oculomotor tracking experiments using a single visual target, control of smooth pursuit movements appears functionally separate from that of the saccadic movements (Rashbass, 1961). Nevertheless, observation of oculomotor behavior in more complex environments shows close temporal association between them; a saccadic shift of fixation is commonly the occasion for a discrete change of pursuit velocity. Different pursuit velocity requirements of successive fixations can result either from the flow of perspective transformations produced by translatory head movements (Gibson, 1950) or from different real velocities of objects. How is the new post-saccade pursuit movement initiated when, e.g., fixation is shifted from a stationary to a moving object?

The visual variable used in controlling smooth pursuit is retinal image velocity (Rashbass, 1961), and the pursuit system's response lag, as determined in tracking experiments, exceeds 80-100 msec (Westheimer, 1954; Rashbass, 1961; Robinson, 1965). The controlling velocity is usually that of the foveal portion of the image. But if fixation is to be shifted to a peripheral moving target, then the increment of pursuit velocity that will be required after completion of the saccadic shift, to stabilize the new target's image with respect to foveal retina, equals the angular velocity with which the same target's image moves across peripheral retina just before the shift. Therefore pre-saccade image velocity information from peripheral retina might well be used for setting post-saccade ocular pursuit velocity. If it were, the pursuit response lag could occur before and during (rather than after) the performance of the saccadic movement, and full stabilization of the new target's image could be achieved more promptly. (It is already known that ocular pursuit movements can be elicited under certain other circumstances by image movement on peripheral retina; e.g., Rademaker \& ter Braak, 1948; Pasik \& Pasik, 1964.)

Method

Eye movements were recorded, using a dc electrooculographic technique (Kris, 1960), while the S looked at a display which included both stationary and moving objects. The S's head was centered just beneath the circular lower rim of a striped, motordriven Barany screen (a vertical cylinder open at the bottom, radius 18 in.; e.g., Pasik \& Pasik, 1964), so that the midpoint between his eyes was in line with the axis of rotation of the screen. If the S's line of gaze was horizontal, then when the screen rotated, the upper portion of his visual field was filled by a series of moving vertical stripes, while the lower portion of his field was occupied by the stationary walls and furnishings of the laboratory. Both screen and laboratory surroundings were diffusely illuminated at normal reading levels. A stationary fixation light was placed directly in front of the $\mathrm{S}, 6-14 \mathrm{in}$. below the rim of the moving screen.

Each test sequence consisted of several fixation shifts, alternately from the stationary to a moving fixation target and then back again; such sequences were repeated using left-to-right and right-to-left rotation of the screen, at angular velocities from 30 to $110 \mathrm{deg} / \mathrm{sec}$.

Two types of tests were conducted: In the first procedure the $S$ shifted his gaze from the stationary light to the moving stripes, then back from the stripes to the light, following the E's directions. This may be called the "verbal signal" procedure.

In the second procedure the $S$ was instructed beforehand in the performance of a visual detection task, in which a small target bearing one of the digits from 1 to 5 swept across his field of gaze at unpredictable intervals. (Height of the number was about 2 deg.) The $E$ clipped the numbered target to the rim of the moving screen at a point directly behind the $S$, and then removed it again after a single revolution; time intervals between target presentations were varied randomly over a range of about 2-5 $\mathrm{sec}$, and the digit shown on the target was also selected at random each time. The $S$ was requested to look at the stationary light until a numbered target came into view, then to note the number on the moving target and to press a key only if it was 5 . This may be called the "vigilance task" procedure.

Four Ss were tested. The first was a neurologically normal young adult male (a member of the laboratory staff); both procedures were used with this $S$. Tests using the "verbal signal" procedure were conducted with three additional Ss (neurologic patients: one male and two female adults), giving results that were qualitatively similar to those obtained with the first $S$. 
(Experiments were terminated at this point by laboratory relocation; details of further experiments, corroborating and extending these initial findings, will be reported elsewhere.)

\section{Results and Discussion}

With either method of testing a constant and consistent pursuit velocity was reached shortly after each fixation shift to a moving target, and was almost always sustained until the $S$ made a new fixation movement to a target with a different velocity: This velocity of sustained pursuit will be referred to as the "final pursuit velocity" of each trial. One-hundred ten eye movements were measured for post-saccade pursuit delay. In more than half of these instances (i.e., 58 of the 110) an appreciable fraction of the final pursuit velocity (i.e., at least 25\%) had been attained within $20 \mathrm{msec}$ after termination of the saccade. In the test in which the "vigilance task" procedure was used, every fixation shift from the stationary to a moving target $(N=26)$ showed this prompt pursuit initiation, and in all but one of these instances the full final pursuit velocity had been reached within $30 \mathrm{msec}$.

If Robinson's (1965) value of $125 \mathrm{msec}( \pm 20 \mathrm{msec}$ SD) for the input-to-output transport delay of the pursuit control system is assumed, it then follows that the sensory information utilized to set the new pursuit velocity impinged upon the retina at least $80 \mathrm{msec}$ before the termination of the saccade. As it is unlikely that the CNS will derive accurate information concerning relative image velocities from the visual input that occurs during the saccade, it seems reasonable to conclude that the pertinent sensory input-or most of it-occurred before the onset of the saccade. These experiments, however, did not set any clear maximum on how long before the saccade the pursuit-controlling input had occurred (since target velocities were changed only occasionally); but similar results have since been obtained in experiments employing brief exposures to targets moving with unpredictable velocities, in which the prompt performance of the appropriate pursuit response must have depended upon velocity information obtained immediately before the fixation shift. This implies utilization of velocity information from that peripheral region of the retina which also determined, by its direction and distance from the fovea, the direction and angular amplitude of the saccadic fixation shift.

In our observations the amplitude of the fixation shifts ranged approximately from 10 to $40 \mathrm{deg}$; thus, inputs from retinal regions at least this far from the fovea can be utilized for initiating pursuit. Furthermore, since (unlike the more usual situation in experiments on ocular pursuit) a profusion both of stationary and of moving contours was always present in the S's field of view during the interval preceding the fixation shift, it appears that the conflicting and redundant velocity information available to the pursuit control system can be utilized in a highly selective manner-though the level at which selection takes place (e.g., retinal, or more central) remains unknown. As the regions of similar visual velocity were fairly large in these initial tests, and no more than two such regions were present at the same time, the extent to which the selective mechanisms may be capable of fine spatial differentiations was not determined; however, recent experiments using 1/2-deg moving targets showed that the selected retinal regions may be of small extent.

\section{References}

Gibson, J. J. The perception of the visual world. Boston: Houghton Mifflin, 1950.

Kris, C. Vision: Electro-oculography. In O. Glasser (Ed.), Medical physics, vol. 3. Chicago: Yearbook Pub. Co., 1960. Pp. 692-700.

Pasik, P., \& Pasik, T. Oculomotor function in monkeys with lesions of the cerebrum and the superior colliculi. In M. B. Bender (Ed.), The oculomotor system. New York: Hoeber, 1964. Pp. 40-80.

Rademaker, G. G. J., \& ter Braak, J. W. G. On the central mechanism of some optic reactions. Brain, 1948, 71, 48-76.

Rashbass, C. The relationship between saccadic and smooth trackIng eye movements. J. Physiol. (London), 1961, 159, 326-338.

Robinson, D. A. The mechanics of human smooth pursuit eye movement. J. Physiol. (London), 1965, 180, 569-591.

Westheimer, G. Eye movement responses to a horizontally moving visual stimulus. Arch. Ophthal., 1954, 52, 932-943.

\section{Note}

1. This work was supported by NIH research grant NB 04576 . 